\title{
DES TROMPEITES PHOTONIQUES pour les technologies quantiques
}

\author{
Jean-Michel GERARD \\ Julien CLAUDON \\ Université Grenoble Alpes, \\ CEA, Institut de Nanoscience et \\ Cryogénie, Grenoble, France \\ jean-michel.gerard@cea.fr \\ julien.claudon@cea.fr
}

La source de photon unique est un composant clef pour le développement des technologies quantiques. À ce jour, les sources les plus performantes exploitent l'émission spontanée d'une boîte quantique semiconductrice, canalisée dans un mode optique bien défıni grâce à une nanostructure photonique. Nous présentons une approche originale, la trompette photonique, qui a permis de réaliser des sources monomodes, efficaces et d'une grande agilité spectrale. Par ailleurs, ces objets possèdent des propriétés optomécaniques remarquables et permettent de réaliser des capteurs ultrasensibles.

fonctionner exactement à la même longueur d'onde dans des réseaux ou simulateurs quantiques. En dépit de leur grande versatilité, les diodes laser monomodes ne remplissent pas tous ces critères : elles

(a)

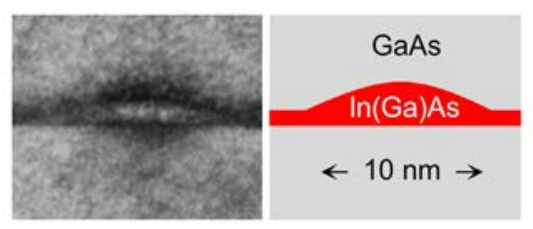

(c)

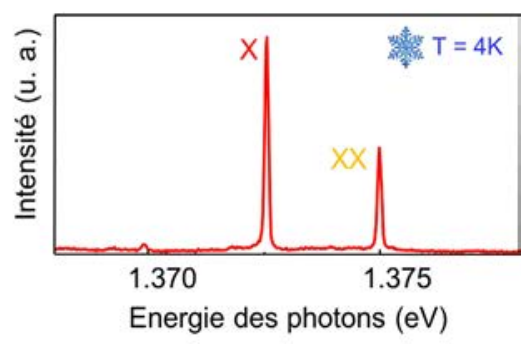

(b)
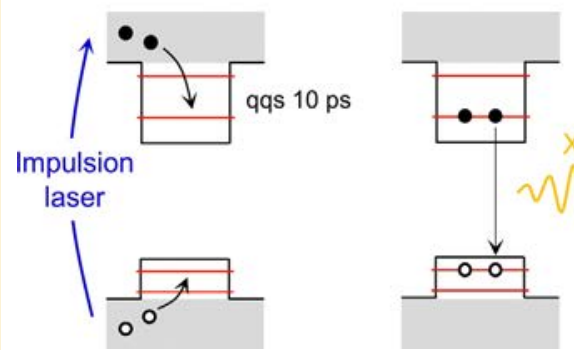

génèrent des états cohérents, pour lesquels la statistique du nombre de photons dans une impulsion suit la loi aléatoire de Poisson, et sont donc intrinsèquement inadaptées à la génération de photon unique. non classiques telles qu'un photon unique ou une paire de photons dans un état quantique enchevêtré. Les domaines d'application potentielle de ces sources se sont diversifiés au fil du temps, pour englober notamment la métrologie (étalon pour la candela) et les simulations quantiques (échantillonnage bosonique).

Les contraintes à satisfaire en vue d'applications réelles sont en pratique très fortes. Une source de photon unique (SPU) doit émettre des impulsions lumineuses à la demande, être pure (pas d'impulsion multiphotonique), efficace (probabilité élevée que les impulsions contiennent effectivement un photon). Dans la plupart des cas, elle doit être monomode, c'est-à-dire que les photons uniques émis successivement doivent être préparés dans le même état quantique (mode spatial, polarisation, paquet d'onde temporel...). Enfin, il est important que les SPUs possèdent une certaine agilité spectrale, de façon à ce que des sources distinctes puissent

Figure 1. Boîtes quantiques et émission de photon unique. (a) Image en microscopie électronique à transmission et coupe schématique illustrant la structure d'une boîte quantique (A. Ponchet, 1995). (b) Emission de photons uniques par une boîte quantique : une impulsion laser excite des paires électron-trou dans le matériau entourant la boîte. Ces dernières sont ensuite capturées rapidement par la boîte. La recombinaison successive de chaque paire électron-trou conduit à l'émission de photons uniques avec des couleurs différentes, qui peuvent être isolés par filtrage spectral. (c) Spectre d'émission typique d'une boîte quantique, mesuré à température cryogénique $(T=4 \mathrm{~K})$. La différence de couleur entre les raies excitonique $(\mathrm{X})$ et biexcitonique (XX) vient de la forte interaction de Coulomb entre porteurs piégés dans la boîte. 
Certaines démonstrations de distribution de clef quantique ont mis en œuvre des impulsions laser très fortement atténuées (0,01 photon en moyenne par impulsion) pour diminuer la proportion d'impulsions multiphotoniques. Cette approche est cependant très pénalisante car elle réduit fortement la portée des liaisons sécurisées. De plus, la violation du critère de pureté limite drastiquement les autres perspectives d'application de ces sources laser atténuées.

Conceptuellement, l'approche la plus simple et la plus élégante pour générer un photon unique consiste à exploiter l'émission spontanée d'un atome isolé. Si on excite un atome de son état fondamental vers son premier état excité, par exemple à l'aide d'une impulsion laser, il retournera spontanément vers son état fondamental en émettant un unique photon. Une variante de ce protocole a par exemple été mise en œuvre par Alain Aspect pour conduire ses fameuses expériences sur la violation des inégalités de Bell. Il est cependant délicat de manipuler un atome isolé et donc intéressant sur un plan pratique de se tourner vers des systèmes quantiques solides présentant, comme un atome, des états électroniques discrets. À la fin des années 90, l'émission d'impulsions à un photon a été démontrée pour de nombreux « atomes artificiels » isolés, molécules, centres colorés du diamant, nanocristaux et boîtes quantiques de semiconducteurs. Au fil du temps, les études se sont concentrées sur les boîtes quantiques (voir figure 1), qui présentent une combinaison unique d'atouts : stabilité dans le temps, capacité à émettre aux longueurs d'onde télécom pour des boîtes de taille adaptée, rendement quantique d'émission proche de l'unité, spectre très fin bien adapté pour les expériences d'optique quantique. Il faut cependant noter que ces deux dernières propriétés sont obtenues au prix d'un fonctionnement à température cryogénique (1-10 K).
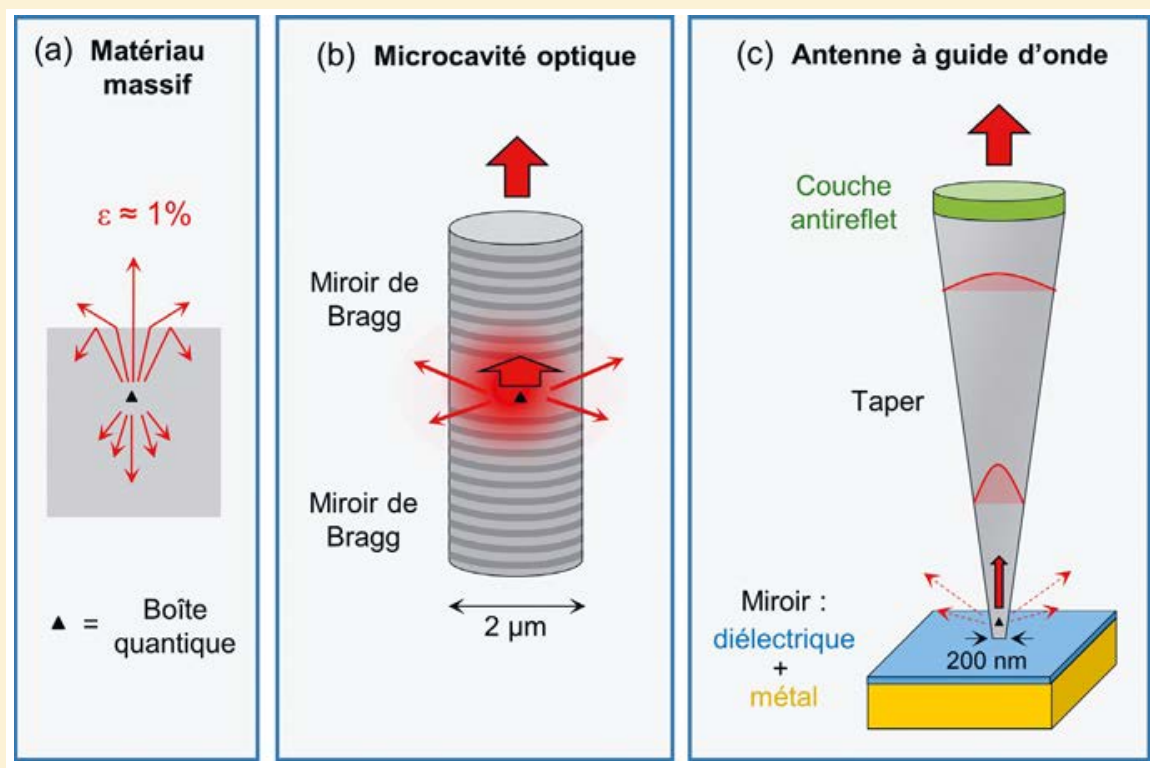

Figure 2. Problématique du contrôle de l'émission spontanée. (a) Si rien n'est fait, la quasi-totalité (99\%) de l'émission de la boîte quantique reste piégée dans la matrice de haut indice de réfraction qui l'entoure. (b) Une microcavité optique, en résonance avec l'émetteur, accélère sélectivement l'émission spontanée dans le mode de cavité (effet Purcell). Les photons sont alors préférentiellement émis dans la cavité. Ils s'échappent ensuite vers le haut, car le miroir supérieur est moins réfléchissant. (c) Antenne optique à guide d'onde ou trompette photonique. La boîte quantique est localisée à la base de la structure, dans un guide d'onde de haut indice. Ce dernier supprime efficacement l'émission dans tous les modes optiques autres que l'unique mode guidé. La collection des photons guidés est optimisée grâce à un miroir idoine, et à un taper qui permet d'obtenir un faisceau directif. Cette approche non résonante est efficace sur une large plage de longueurs d'ondes.

\section{Une source de photon unique efficace à trompette photonique}

Choisir un bon atome artificiel n'est cependant pas suffisant pour fabriquer une SPU monomode et efficace. En effet, de façon habituelle, l'émission spontanée tend à être rayonnée dans toutes les directions de l'espace. En vue d'une application pratique, il faut rendre le diagramme de rayonnement aussi directionnel que possible ou, mieux encore, canaliser toute l'émission dans un mode bien défini du champ électromagnétique. Ce point est particulièrement crucial pour les boîtes quantiques, qui sont, du fait de leur mode de fabrication, intégrées au sein d'un matériau de fort indice de réfraction (figure 2a). Ainsi, une part prépondérante de l'émission de la boîte quantique reste piégée dans le matériau, par réflexion totale interne. Pour réaliser une SPU efficace et monomode, il est impératif de « modeler » l'émission spontanée. Les concepts de l'électrodynamique quantique en cavité, développée par Serge Haroche et ses collègues, sont une puissante ressource dans ce contexte.

En 2001, le Laboratoire de Photonique et de Nanostructures (LPN) du CNRS (actuellement Centre de Nanosciences et de Nanotechnologies, C2N) a présenté la première source de photons uniques monomodes, obtenue en plaçant une boite quantique au cœur d'une microcavité optique en forme de micropilier [1]. La boîte quantique est placée en accord avec le mode résonant fondamental du micropilier, afin d'entrer dans un état connu sous le nom d'effet Purcell. L'émission de la boîte quantique dans ce mode particulier est alors très fortement exaltée, ce qui a deux conséquences majeures. D’une part, l'émission du photon unique par la boîte devient de 5 à 100 fois plus rapide. D'autre part, presque toute l'émission spontanée de la boîte est canalisée dans le mode résonant de la cavité, du fait du caractère sélectif de l'effet Purcell (figure 2b). 


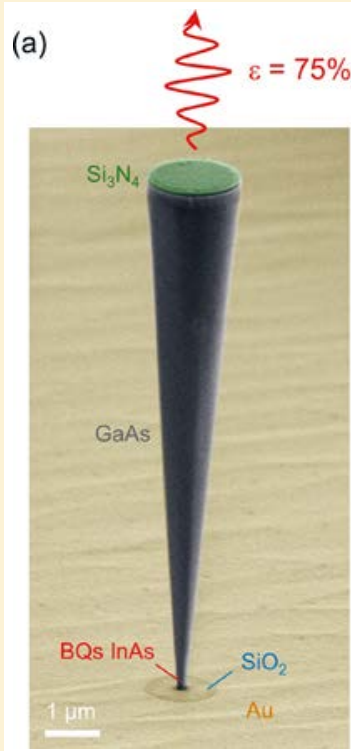

(b)

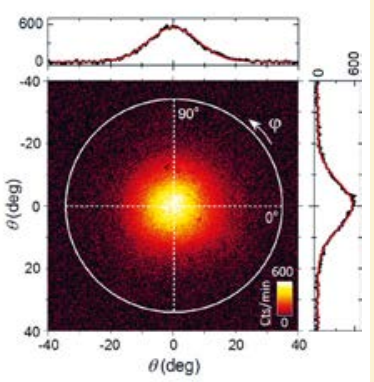

(c)

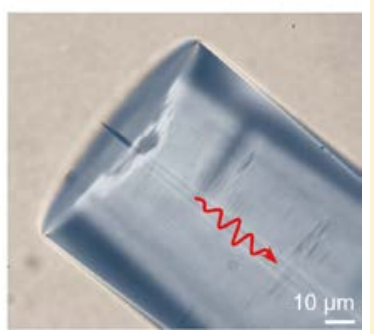

Figure 3. Une source de photons uniques très efficace. (a) Vue en microscopie électronique d'une trompette photonique (fausses couleurs). Grâce à cette nanostructure photonique, 75 \% des photons émis par une boîte quantique peuvent être collectés par un objectif de microscope. (b) Profil angulaire du faisceau émis par l'antenne. La mesure, réalisée dans le plan de Fourier, révèle un profil transverse parfaitement Gaussien. (c) Trompette photonique détachée de son substrat et collée sur la face clivée d'une fibre optique monomode (image en microscopie optique). Les photons sont émis directement dans le mode guidé par la fibre ; ce dispositif intégré ne nécessite pas d'alignement optique.

Plusieurs équipes, notamment celle de Pascale Senellart au C2N et celle de Sven Höfling à l'université de Würzburg, ont poussé cette approche jusqu'à un haut niveau de perfection, et présenté des SPUs combinant un haut niveau de pureté, une bonne indiscernabilité des photons émis, et une efficacité de l'ordre de 0,7 photon par impulsion. La mise en œuvre de l'effet Purcell, qui impose l'accord spectral entre la boîte quantique et un mode résonant de haut facteur de qualité $Q$, complique la fabrication de ces SPUs et limite fortement leur plage d'accordabilité spectrale. En outre, il est bien connu que le diagramme de rayonnement de microcavités de grand $Q$ est très sensible aux imperfections de fabrication, telles que la rugosité des flancs gravés des micropiliers. En dépit des remarquables progrès technologiques réalisés depuis 2001, cette difficulté est un obstacle au développement de SPU d'efficacité ultime (> 0,99).

Pour contourner ces problèmes, l'Institut de Nanoscience et de Cryogénie a récemment introduit une nouvelle géométrie pour les SPU, la trompette photonique (figure 2c) [2,3], dont le développement a été récompensé par une médaille de Bronze du CNRS en 2014. La boîte quantique est intégrée dans un nanofil en GaAs, défini par gravure en salle blanche, qui forme un guide d'onde en forme de cône. Du fait du fort contraste d'indice entre GaAs et l'air, un effet d'écrantage diélectrique inhibe fortement l'émission spontanée de la boîte dans l'espace libre environnant. Des études théoriques montrent que, de ce fait, $95 \%$ de l'émission de la boîte est injectée dans le mode guidé du fil photonique. Un miroir adapté est placé au pied de la trompette pour rediriger toute l'émission vers le haut. La forme évasée du fil photonique permet d'augmenter de façon adiabatique la taille latérale du mode guidé, pour obtenir un diagramme de rayonnement Gaussien faiblement divergent, bien adapté par exemple au couplage direct à une fibre optique en vue d'une application aux communications quantiques (figure 3). Les trompettes photoniques ont permis de réaliser des SPU très pures, et de haute efficacité ( 0,75 photon/impulsion). De plus, il est possible de modifier la longueur d'onde de l'émetteur sans dégrader l'efficacité de la SPU. En collaboration avec l'équipe de J.P. Poizat de l'Institut Néel (Grenoble), nous avons démontré récemment une source accordable sur une plage spectrale remarquablement large $(25 \mathrm{~nm})$, en appliquant une contrainte mécanique à la boîte quantique.

\section{Un système optomécanique hybride pouri les capteuirs quantiques}

Les trompettes photoniques possèdent par ailleurs des caractéristiques mécaniques remarquables et constituent un nouveau type de système optomécanique hybride, qui associe un atome artificiel (ici la boîte quantique) et un oscillateur mécanique [4]. En effet, lorsque le fil vibre, sa base est soumise à une contrainte qui oscille entre compression et extension. Il en résulte une forte modulation de l'énergie de bande interdite de la boîte quantique qui est directement visible sur un spectre de photoluminescence (figure 4b), comme le montrent les expériences menées en collaboration avec des collègues de l'Institut Néel (Grenoble). La recherche autour des systèmes hybrides est actuellement en pleine effervescence. Une des motivations principales qui anime le domaine est de transférer le caractère quantique de l'atome artificiel à l'oscillateur mécanique, pour explorer la frontière entre le monde quantique microscopique et le monde classique macroscopique. Au-delà de cet objectif fondamental très ambitieux, et dans une perspective plus appliquée, les systèmes hybrides offrent une voie originale pour réaliser des capteurs quantiques performants.

Dans le cadre d'une collaboration avec l'Université de Bâle (Suisse), nous avons ainsi utilisé la boîte quantique comme sonde très sensible du déplacement du fil [5].

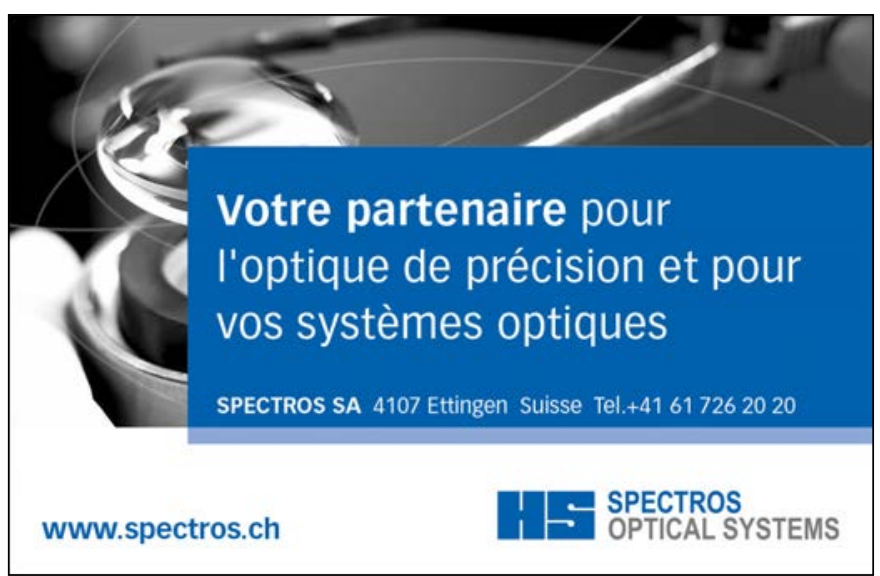


(a)

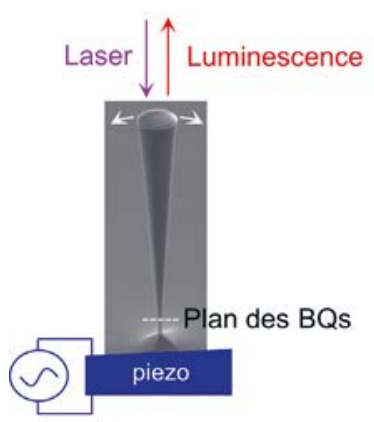

(c)

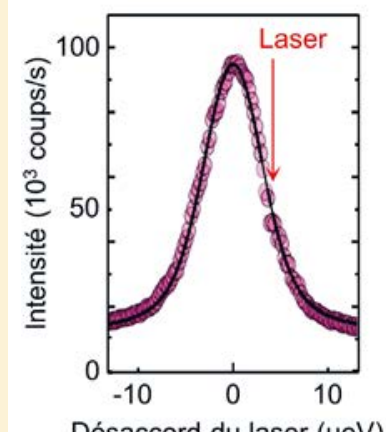

(b)

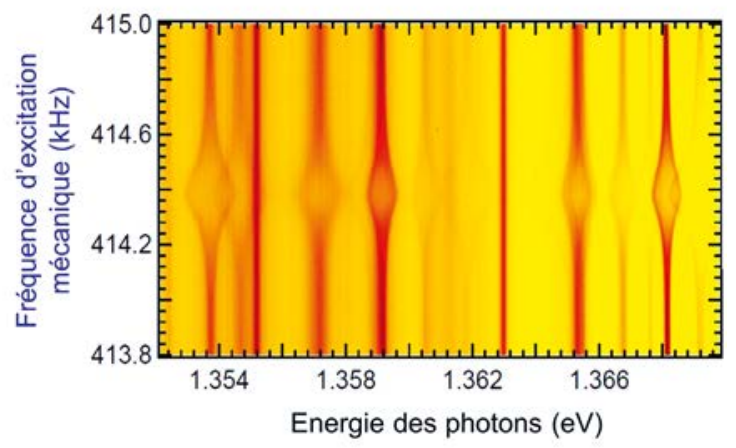

(d)

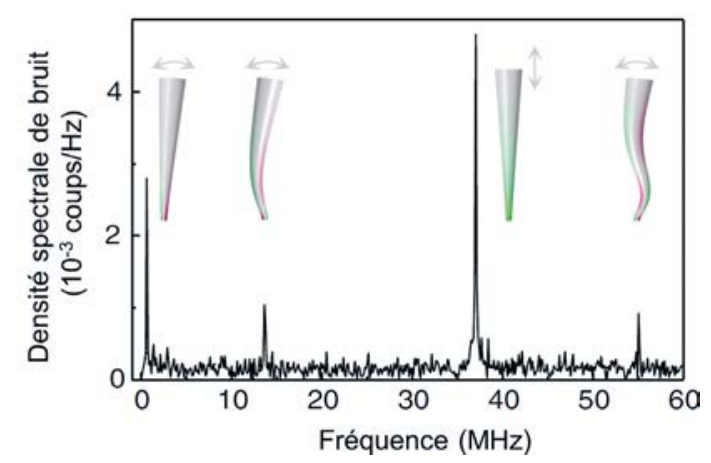

Figure 4. Une nouvelle plateforme pour l'optomécanique hybride. (a) Le mode fondamental de vibration du fil, qui correspond à une flexion autour de la base, est excité grâce à un actuateur piézoélectrique. On excite simultanément la luminescence de toutes les boîtes quantiques intégrées au pied de la structure avec un laser continu. (b) Spectre de photoluminescence des boîtes sous excitation mécanique. La couleur code l'intensité de la luminescence (rouge $=$ forte, jaune $=$ faible). Lorsqu'un mode de vibration est excité de manière résonante (ici à $414,4 \mathrm{kHz}$ ), le gap de la boîte quantique oscille en phase avec l'oscillation mécanique, avec une amplitude qui dépend fortement de sa position au sein du fil. (c) Pour détecter les vibrations mécaniques d'un fil au repos avec une grande sensibilité, on accorde un laser spectralement fin sur le flanc de la raie spectrale associée à une boîte quantique. Toute fluctuation de gap modifie le pompage de la boîte et l'intensité du signal de fluorescence résonante. (d) Spectre mécanique obtenu par transformée de Fourier du signal collecté. La technique est très sensible : on résout ici très facilement les vibrations thermiques du fil, même à température cryogénique ( $T=4 \mathrm{~K})$.

Pour ce faire, un laser spectralement fin est accordé sur le flanc de la résonance optique associée à une boîte (figure 4c). Toute vibration du fil induit alors un décalage spectral de la transition et modifie donc l'intensité du signal émis par la boîte quantique. La technique est tellement sensible qu'elle permet de résoudre les vibrations thermiques du fil à température cryogénique (figure 4d), soit des amplitudes de vibration de l'ordre de la centaine de femtomètres, qui correspondent à des déformations infimes de la maille cristalline $\left(10^{-8} \mathrm{en}\right.$ valeur relative).

De manière symétrique, nous avons également montré avec nos collègues grenoblois que la contrainte mécanique permet de localiser finement les boîtes quantiques intégrées dans le fil. En inspectant en détail la figure $4 b$, on constate en effet que la réponse à l'oscillation mécanique présente de fortes variations d'une boîte à l'autre. Ceci s'explique par la répartition très inhomogène de la contrainte dans le plan des boîtes quantiques. Lorsque le fil est tordu, la contrainte présente un axe neutre qui sépare une zone en extension d'une zone en compression. Plus on s'éloigne de l'axe neutre et plus la contrainte est forte en valeur absolue. De manière tout à fait analogue à l'imagerie par résonance magnétique, l'immersion des boîtes dans ce fort gradient de contrainte permet de cartographier leur position avec une grande précision (jusqu'à $1 \mathrm{~nm}$ ) [6].

\section{Perspectives}

Pour conclure, les trompettes photoniques constituent une plate-forme versatile pour l'optique quantique. Grâce à leur large bande passante, elles sont en particulier très adaptées à la réalisation de sources brillantes de photons enchevêtrés, un composant clé pour les communications quantiques à longue distance. Par ailleurs, le fort couplage optomécanique permettra de manipuler l'état de vibration de la trompette via une excitation optique de la boîte quantique (1000 fois plus petite qu'elle !), afin de générer des états mécaniques non classiques.

\section{POUR EN SAVOIR PLUS}

[1] E. Moreau, I. Robert, J.M. Gérard, I. Abram, L. Manin, V. Thierry-Mieg, Single-mode solid-state single photon source based on isolated quantum dots in pillar microcavities, Appl. Phys. Lett. 79, 2865 (2001)

[2] J. Claudon, J. Bleuse, N.S. Malik, M. Bazin, P. Jaffrennou, N. Gregersen, C. Sauvan, P. Lalanne, J.-M. Gérard, A highly efficient single-photon source based on a quantum dot in a photonic nanowire, Nature Photon. 4, 174 (2010)

[3] M. Munsch, N.S. Malik, E. Dupuy, A. Delga, J. Bleuse, J.-M. Gérard, J. Claudon, N. Gregersen, J. Mørk, Dielectric GaAs antenna ensuring an efficient broadband coupling between an InAs quantum dot and a Gaussian optical beam, Phys. Rev. Lett. 110, 177402 (2013)

[4] I. Yeo, P.-L. de Assis, A. Gloppe, E. Dupont-Ferrier, P. Verlot, N.S. Malik, E. Dupuy, J. Claudon, J.-M. Gérard, A. Auffeves, G. Nogues, S. Seidelin, J.-Ph. Poizat, O. Arcizet, M. Richard, Strain-mediated coupling in a quantum dot-mechanical oscillator hybrid system, Nature Nanotech. 9, 106 (2014)

[5] M. Munsch, A.V. Kuhlmann, D. Cadeddu, J.-M. Gérard, J. Claudon, M. Poggio, R. J. Warburton, Resonant driving of a single photon emitter embedded in a mechanical oscillator, Nat. Commun. 8 76 (2017)

[6] P.-L. De Assis, I. Yeo, A. Gloppe, H.A. Nguyen, D. Tumanov, E. DupontFerrier, N.S. Malik, E. Dupuy, J. Claudon, J.-M Gérard, A. Auffeves, O. Arcizet, M. Richard, J.-Ph. Poizat, Strain-gradient position mapping of semiconductor quantum dots, Phys. Rev. Lett. 118, 117401 (2017) 